\title{
Some non-normal Cayley digraphs of the generalized quaternion group of certain orders
}

\author{
Edward Dobson \\ Department of Mathematics and Statistics \\ PO Drawer MA \\ Mississippi State, MS 39762, U.S.A. \\ dobson@math.msstate.edu
}

Submitted: Mar 10, 2003; Accepted: Jul 30, 2003; Published: Sep 8, 2003

MR Subject Classifications: 05C25, 20B25

\begin{abstract}
We show that an action of $\mathrm{SL}(2, p), p \geq 7$ an odd prime such that $4 \backslash(p-1)$, has exactly two orbital digraphs $\Gamma_{1}, \Gamma_{2}$, such that $\operatorname{Aut}\left(\Gamma_{i}\right)$ admits a complete block system $\mathcal{B}$ of $p+1$ blocks of size $2, i=1,2$, with the following properties: the action of $\operatorname{Aut}\left(\Gamma_{i}\right)$ on the blocks of $\mathcal{B}$ is nonsolvable, doubly-transitive, but not a symmetric group, and the subgroup of $\operatorname{Aut}\left(\Gamma_{i}\right)$ that fixes each block of $\mathcal{B}$ set-wise is semiregular of order 2. If $p=2^{k}-1>7$ is a Mersenne prime, these digraphs are also Cayley digraphs of the generalized quaternion group of order $2^{k+1}$. In this case, these digraphs are non-normal Cayley digraphs of the generalized quaternion group of order $2^{k+1}$.
\end{abstract}

There are a variety of problems on vertex-transitive digraphs where a natural approach is to proceed by induction on the number of (not necessarily distinct) prime factors of the order of the graph. For example, the Cayley isomorphism problem (see [6]) is one such problem, as well as determining the full automorphism group of a vertex-transitive digraph $\Gamma$. Many such arguments begin by finding a complete block system $\mathcal{B}$ of $\operatorname{Aut}(\Gamma)$. Ideally, one would then apply the induction hypothesis to the groups $\operatorname{Aut}(\Gamma) / \mathcal{B}$ and $\left.\operatorname{fix}_{\operatorname{Aut}(\Gamma)}(\mathcal{B})\right|_{B}$, where $\operatorname{Aut}(\Gamma) / \mathcal{B}$ is the permutation group induced by the action of $\operatorname{Aut}(\Gamma)$ on $\mathcal{B}$, and $\operatorname{fix}_{\operatorname{Aut}(\Gamma)}(\mathcal{B})$ is the subgroup of $\operatorname{Aut}(\Gamma)$ that fixes each block of $\mathcal{B}$ set-wise, and $B \in \mathcal{B}$. Unfortunately, neither $\operatorname{Aut}(\Gamma) / \mathcal{B}$ nor $\left.\operatorname{fix}_{\operatorname{Aut}(\Gamma)}(\mathcal{B})\right|_{B}$ need be the automorphism group of a digraph. In fact, there are examples of vertex-transitive graphs where $\operatorname{Aut}(\Gamma) / \mathcal{B}$ is a doubly-transitive nonsolvable group that is not a symmetric group (see [7]), as well as examples of vertex-transitive graphs where $\left.\operatorname{fix}_{\operatorname{Aut}(\Gamma)}(\mathcal{B})\right|_{B}$ is a doubly-transitive nonsolvable group that is not a symmetric group (see [2]). (There are also examples where $\operatorname{Aut}(\Gamma) / \mathcal{B}$ is a solvable doubly-transitive group, but in practice, this is not usually 
a genuine obstacle in proceeding by induction.) The only known class of examples of vertex-transitive graphs where $\operatorname{Aut}(\Gamma) / \mathcal{B}$ is a doubly-transitive nonsolvable group, have the property that $\operatorname{Aut}(\Gamma) / \mathcal{B}$ is a faithful representation of $\operatorname{Aut}(\Gamma)$ and $\Gamma$ is not a Cayley graph. In this paper, we give examples of vertex-transitive digraphs that are Cayley digraphs and the action of $\operatorname{Aut}(\Gamma) / \mathcal{B}$ on $\mathcal{B}$ is doubly-transitive, nonsolvable, not faithful, and not a symmetric group.

\section{Preliminaries}

Definition 1.1 Let $G$ be a permutation group acting on $\Omega$. If $\omega \in \Omega$, then a sub-orbit of $G$ is an orbit of $\operatorname{Stab}_{G}(\omega)$.

Definition 1.2 Let $G$ be a finite group. The socle of $G$, denoted $\operatorname{soc}(G)$, is the product of all minimal normal subgroups of $G$. If $G$ is primitive on $\Omega$ but not doubly-transitive, we say $G$ is simply primitive. Let $G$ be a transitive permutation group on a set $\Omega$ and let $G$ act on $\Omega \times \Omega$ by $g(\alpha, \beta)=(g(\alpha), g(\beta))$. The orbits of $G$ in $\Omega \times \Omega$ are called the orbitals of $G$. The orbit $\{(\alpha, \alpha): \alpha \in \Omega\}$ is called the trivial orbital. Let $\Delta$ be an orbital of $G$ in $\Omega \times \Omega$. Define the orbital digraph $\Delta$ to be the graph with vertex set $\Omega$ and edge set $\Delta$. Each orbital of $G$ has a paired orbital $\Delta^{\prime}=\{(\beta, \alpha):(\alpha, \beta) \in \Delta\}$. Define the orbital graph $\Delta$ to be the graph with vertex set $\Omega$ and edge set $\Delta \cup \Delta^{\prime}$. Note that there is a canonical bijection from the set of orbital digraphs of $G$ to the set of sub-orbits of $G$ (for fixed $\omega \in \Omega$ ).

Definition 1.3 Let $G$ be a transitive permutation group of degree $m k$ that admits a complete block system $\mathcal{B}$ of $m$ blocks of size $k$. If $g \in G$, then $g$ permutes the $m$ blocks of $\mathcal{B}$ and hence induces a permutation in $S_{m}$, which we denote by $g / \mathcal{B}$. We define $G / \mathcal{B}=\{g / \mathcal{B}: g \in G\}$. Let $\operatorname{fix}_{\mathcal{B}}(G)=\{g \in G: g(B)=B$ for every $B \in \mathcal{B}\}$.

Definition 1.4 Let $G$ be transitive group acting on $\Omega$ with $r$ orbital digraphs $\Gamma_{1}, \ldots, \Gamma_{r}$. Define the 2-closure of $G$, denoted $G^{(2)}$ to be $\cap_{i=1}^{r} \operatorname{Aut}\left(\Gamma_{i}\right)$. Note that if $G$ is the automorphism group of a vertex-transitive digraph, then $G^{(2)}=G$.

Definition 1.5 Let $\Gamma$ be a graph. Define the complement of $\Gamma$, denoted by $\bar{\Gamma}$, to be the graph with $V(\bar{\Gamma})=V(\Gamma)$ and $E(\bar{\Gamma})=\{u v: u, v \in V(\Gamma)$ and $u v \notin E(\Gamma)\}$.

Definition 1.6 A group $G$ given by the defining relations

$$
G=\left\langle h, k: h^{2^{a-1}}=k^{2}=m, m^{2}=1, k^{-1} h k=h^{-1}\right\rangle
$$

is a generalized quaternion group.

Let $p \geq 5$ be an odd prime. Then $\operatorname{GL}(2, p)$ acts on the set $\mathbb{F}_{p}^{2}$, where $\mathbb{F}_{p}$ is the field of order $p$, in the usual way. This action has two orbits, namely $\{0\}$ and $\Omega=\mathbb{F}_{p}^{2}-\{0\}$. The action of $\operatorname{GL}(2, p)$ on $\Omega$ is imprimitive, with a complete block system $\mathcal{C}$ of $\left(p^{2}-1\right) /(p-1)=$ $p+1$ blocks of size $p-1$, where the blocks of $\mathcal{C}$ consist of all scalar multiples of a given 
vector in $\Omega$ (these blocks are usually called projective points), and the action of GL $(2, p)$ on the blocks of $\mathcal{C}$ is doubly-transitive. Furthermore, $\operatorname{fix}_{\mathrm{GL}(2, p)}(\mathcal{C})$ is cyclic of order $p-1$, and consists of all scalar matrices $\alpha I$ (where $I$ is the $2 \times 2$ identity matrix) in GL $(2, p)$. Note that if $m \mid(p-1)$, then $\operatorname{GL}(2, p)$ admits a complete block system $\mathcal{C}_{m}$ of $(p+1) m$ blocks of size $(p-1) / m$, and fix $\mathrm{GL}_{(2, p)}\left(\mathcal{C}_{m}\right)$ consists of all scalar matrices $\alpha^{i} I$, where $\alpha \in \mathbb{F}_{p}^{*}$ is of order $(p-1) / m$ and $i \in \mathbb{Z}$. Each such block of $\mathcal{C}_{m}$ consists of all scalar multiples $\alpha^{i} v$, where $v$ is a vector in $\mathbb{F}_{p}^{2}$ and $i \in \mathbb{Z}$. Hence $\operatorname{GL}(2, p) / \mathcal{C}_{m}$ admits a complete block system $\mathcal{D}_{m}$ consisting of $p+1$ blocks of size $m$, induced by $\mathcal{C}_{m}$. Henceforth, we set $m=2$ so that $\mathcal{C}_{2}$ consists of $2(p+1)$ blocks of size $(p-1) / 2$, and $\mathcal{D}_{2}$ consists of $p+1$ blocks of size 2 . Note that as $p \geq 5, \operatorname{SL}(2, p)$ is doubly-transitive on the set of projective points, as if $A \in \mathrm{GL}(2, p)$, then $\operatorname{det}(A)^{-1} A \in \mathrm{SL}(2, p)$. Finally, observe that $(-1) I \in \mathrm{SL}(2, p)$. Thus $(-1) I / \mathcal{C}_{2} \in \operatorname{fix} \operatorname{SL}(2, p) / \mathcal{C}_{2}\left(\mathcal{D}_{2}\right) \neq 1$ so that $\mathrm{SL}(2, p) / \mathcal{C}_{2}$ is transitive on $\mathcal{C}_{2}$. Additionally, as $\operatorname{fix}_{\mathrm{GL}(2, p)}\left(\mathcal{C}_{2}\right)=\left\{\alpha^{i} I:|\alpha|=(p-1) / 2, i \in \mathbb{Z}\right\}, \operatorname{SL}(2, p) / \mathcal{C}_{2} \cong \operatorname{SL}(2, p)$. That is, $\operatorname{SL}(2, p) / \mathcal{C}_{2}$ is a faithful representation of $\mathrm{SL}(2, p)$. We will thus lose no generality by referring to an element $x / \mathcal{C}_{2} \in \mathrm{SL}(2, p) / \mathcal{C}_{2}$ as simply $x \in \mathrm{SL}(2, p)$. As each projective point can be written as a union of two blocks contained in $\mathcal{C}_{2}$, we will henceforth refer to blocks in $\mathcal{C}_{2}$ as projective half-points.

\section{Results}

We begin with a preliminary result.

Lemma 2.1 Let $p \geq 7$ be an odd prime such that 4 X $(p-1)$, and let $\mathrm{SL}(2, p)$ act as above on the $2(p+1)$ projective half-points. Then the following are true:

1. $\mathrm{SL}(2, p)$ has exactly four sub-orbits; two of size 1 and 2 of size $p$,

2. $\mathrm{SL}(2, p)$ admits exactly one non-trivial complete block system which consists of $p+1$ blocks of size 2 , namely $\mathcal{D}_{2}$, formed by the orbits of $(-1) I$.

Proof. By [4, Theorem 2.8.1], $|\mathrm{SL}(2, p)|=\left(p^{2}-1\right) p$. It was established above that $\operatorname{SL}(2, p)$ admits $\mathcal{D}_{2}$ as a complete block system of $p+1$ blocks of size 2 , and this complete block system is formed by the orbits of $(-1) I$ as $(-1) I \in \operatorname{fix}_{\mathrm{SL}(2, p)}\left(\mathcal{D}_{2}\right)$ and is semi-regular of order 2. As $\operatorname{SL}(2, p) / \mathcal{D}_{2}=\operatorname{PSL}(2, p)$ is doubly-transitive, there are two sub-orbits of $\operatorname{SL}(2, p) / \mathcal{D}_{2}$, one of size 1 and the other of size $p$. Now, consider $\operatorname{Stab}_{\mathrm{SL}(2, p)}(x)$, where $x$ is a projective half-point. Then there exists another projective half-point $y$ such that $x \cup y$ is a projective point $z$. As $\{x, y\} \in \mathcal{D}_{2}$ is a block of size 2 of $\mathrm{SL}(2, p)$, we have that $\operatorname{Stab}_{\mathrm{SL}(2, p)}(x)=\operatorname{Stab}_{\mathrm{SL}(2, p)}(y)$. Thus $\mathrm{SL}(2, p)$ has at least two singleton sub-orbits. As $\operatorname{SL}(2, p) / \mathcal{D}_{2}=\operatorname{PSL}(2, p)$ has one singleton sub-orbit, $\operatorname{SL}(2, p)$ has exactly two singleton sub-orbits. We conclude that every non-singleton sub-orbit of $\operatorname{SL}(2, p)$ has order a multiple of $p$. As the non-singleton sub-orbits of $\mathrm{SL}(2, p)$ have order a multiple of $p, \operatorname{Stab}_{\mathrm{SL}(2, p)}(x)$ has either one non-singleton orbit of size $2 p$ or two non-singleton orbits of size $p$. As the order of a non-singleton orbit must divide $\left|\operatorname{Stab}_{\mathrm{SL}(2, p)}(x)\right|=p(p-1) / 2$ which is odd as 
$4 \nmid(p-1), \operatorname{SL}(2, p)$ must have exactly two non-singleton sub-orbits of size $p$. Thus 1$)$ follows.

Suppose that $\mathcal{D}$ is another non-trivial complete block system of $\operatorname{SL}(2, p)$. Let $D \in \mathcal{D}$ with $v$ a projective half-point in $D$. By [3, Exercise 1.5.9], $D$ is a union of orbits of $\operatorname{Stab}_{\mathrm{SL}(2, p)}(v)$, so that $|D|$ is either $2, p+1, p+2,2 p$, or $2 p+1$. Furthermore, as the size of a block of a permutation group divides the degree of the permutation group, $|D|=2$ or $p+1$. If $|D|=2$, then $D$ is the union of two singleton orbits of $\operatorname{Stab}_{\mathrm{SL}(2, p)}(v)$, in which case $D$ consists of two projective half-points whose union is a projective point. Thus if $|D|=2$, then $D \in \mathcal{D}_{2}$ and $\mathcal{D}=\mathcal{D}_{2}$. If $|D|=p+1$, then $\mathcal{D}$ consists of 2 blocks of size $p+1$ and $D$ is the union of two orbits of $\operatorname{Stab}_{\mathrm{SL}(2, p)}(v)$, and these orbits have size 1 and $p$. We conclude that $\cup D$ does not contain the projective point $q$ that contains $v$.

Now, $\operatorname{fix}_{\mathrm{SL}(2, p)}(\mathcal{D})$ cannot be trivial, as $\mathrm{SL}(2, p) / \mathcal{D}$ is of degree 2 while $|\mathrm{SL}(2, p)|=$ $\left(p^{2}-1\right) p$. Then $\left|\operatorname{fix}_{\mathrm{SL}(2, p)}(\mathcal{D})\right|=\left(p^{2}-1\right) p / 2$ as $\mathrm{SL}(2, p) / \mathcal{D}$ is a transitive subgroup of $S_{2}$. Furthermore, $-I \notin$ fix $_{\mathrm{SL}(2, p)}(\mathcal{D})$ as no block of $\mathcal{D}$ contains the projective point $q$ that contains $v$ so that $-I$ permutes the two projective half-points whose union is $q$. Thus $\operatorname{fix}_{\mathrm{SL}(2, p)}\left(\mathcal{D}_{2}\right) \cap \operatorname{fix}_{\mathrm{SL}(2, p)}(\mathcal{D})=1$. As $\langle-I\rangle=\operatorname{fix}_{\mathrm{SL}(2, p)}\left(\mathcal{D}_{2}\right)$ and both $\operatorname{fix}_{\mathrm{SL}(2, p)}\left(\mathcal{D}_{2}\right)$ and fix $_{\mathrm{SL}(2, p)}(\mathcal{D})$ are normal in $\mathrm{SL}(2, p)$, we have that $\mathrm{SL}(2, p)=\operatorname{fix}_{\mathrm{SL}(2, p)}\left(\mathcal{D}_{2}\right) \times \operatorname{fix}_{\mathrm{SL}(2, p)}(\mathcal{D})$. Thus a Sylow 2-subgroup of $\mathrm{SL}(2, p)$ can be written as a direct product of two nontrivial 2-groups, contradicting [4, Theorem 8.3].

Theorem 2.2 Let $p \geq 7$ be an odd prime such that 4 X $(p-1)$. Then there exist exactly two digraphs $\Gamma_{i}, i=1,2$ of order $2(p+1)$ such that the following properties hold:

1. $\Gamma_{i}$ is an orbital digraph of $\mathrm{SL}(2, p)$ in its action on the set of projective half-points and is not a graph,

2. $\operatorname{Aut}\left(\Gamma_{i}\right)$ admits a unique nontrivial complete block system $\mathcal{D}_{2}$ which consists of $p+1$ blocks of size 2 ,

3. $\operatorname{fix}_{\mathrm{Aut}\left(\Gamma_{i}\right)}\left(\mathcal{D}_{2}\right)=\langle-I\rangle$ is cyclic of order 2 ,

4. $\operatorname{soc}\left(\operatorname{Aut}\left(\Gamma_{i}\right) / \mathcal{D}_{2}\right)$ is doubly-transitive but $\operatorname{soc}\left(\operatorname{Aut}\left(\Gamma_{i}\right) / \mathcal{D}_{2}\right) \neq A_{p+1}$.

ProOF. By Lemma 2.1, SL $(2, p)$ in its action on the half-projective points has exactly four orbital digraphs; one consisting of $p+1$ independent edges (the edges of this graph consists of all edges of the form $(v, w)$, where $\cup\{v, w\}$ is a projective point; thus $\cup\{v, w\}$ is a block of $\mathcal{D}_{2}$ ), one which consists of only self-loops (and so is trivial with automorphism group $S_{2 p+2}$ and will henceforth be ignored) and two in which each vertex has in and out degree $p$. The orbital digraph $\Gamma$ of $\mathrm{SL}(2, p)$ consisting of $p+1$ independent edges is then $\bar{K}_{p+1} \prec K_{2}$. The other orbital digraphs of $\operatorname{SL}(2, p)$, say $\Gamma_{1}$ and $\Gamma_{2}$, each have in-degree and out-degree $p$.

If either $\Gamma_{1}$ or $\Gamma_{2}$ is a graph, then assume without loss of generality that $\Gamma_{1}$ is a graph. Then whenever $(a, b) \in E\left(\Gamma_{1}\right)$ then $(b, a) \in E\left(\Gamma_{1}\right)$. As $\Gamma_{1}$ is an orbital digraph, there exists $\alpha \in \mathrm{SL}(2, p)$ such that $\alpha(a)=b$ and $\alpha(b)=a$. Raising $\alpha$ to an appropriate odd 
power, we may assume that $\alpha$ has order a power of 2 , and so $\alpha \in Q$, where $Q$ is a Sylow 2-subgroup of $\mathrm{SL}(2, p)$. As a Sylow 2-subgroup of $\mathrm{SL}(2, p)$ is isomorphic to a generalized quaternion by [4, Theorem 8.3], $Q$ contains a unique subgroup of order 2 (see [4, pg. 29]), which is necessarily $\langle-I\rangle$. If $\alpha$ is not of order 2 , then $\alpha^{2}(a)=a$ and $\alpha^{2}(b)=b$ so that $\alpha$ has at least two fixed points. However, $\left(\alpha^{2}\right)^{c}=-I$ for some $c \in \mathbb{Z}$ and $-I$ has no fixed points, a contradiction. Thus $\alpha$ has order 2 and so $\alpha=-I$. Thus $(a, b) \in \bar{K}_{p+1} \prec K_{2} \neq \Gamma_{1}$, a contradiction. Hence 1) holds.

We now establish that 2) holds. Suppose that for $i=1$ or $2, \operatorname{Aut}\left(\Gamma_{i}\right)$ is primitive. We may then assume without loss of generality that $\operatorname{Aut}\left(\Gamma_{1}\right)$ is primitive, and as $\operatorname{Aut}\left(\Gamma_{1}\right) \neq$ $K_{2(p+1)}$, Aut $\left(\Gamma_{1}\right)$ is simply primitive, and, of course, $\operatorname{SL}(2, p)^{(2)} \leq \operatorname{Aut}\left(\Gamma_{1}\right)$. First observe that by [11, Theorem 4.11], $\operatorname{SL}(2, p)^{(2)}$ admits $\mathcal{D}_{2}$ as a complete block system. Let $v$ be a projective half-point. By Lemma $2.1, \operatorname{SL}(2, p)$ has four sub-orbits relative to $v$, two of size 1 , say $\mathcal{O}_{1}=\{v\}$ and $\mathcal{O}_{2}=\{w\}$, and two of size $p$, say $\mathcal{O}_{3}$ and $\mathcal{O}_{4}$. By [11, Theorem 5.5 (ii)] the sub-orbits of $\mathrm{SL}(2, p)^{(2)}$ relative to $v$ are the same as the sub-orbits of $\operatorname{SL}(2, p)$ relative to $v$. Thus the neighbors of $v$ in $\Gamma_{1}$ consist of all elements in one of the sub-orbits $\mathcal{O}_{3}$ or $\mathcal{O}_{4}$. Without loss of generality, assume that this sub-orbit is $\mathcal{O}_{3}$. As $\operatorname{Aut}\left(\Gamma_{1}\right)$ is primitive, by [3, Theorem 3.2A], every non-trivial orbital digraph of $\operatorname{Aut}\left(\Gamma_{1}\right)$ is connected. Then the orbital digraph of $\operatorname{Aut}\left(\Gamma_{1}\right)$ that contains $v \vec{w}$ is connected, and so $\mathcal{O}_{2}=\{w\}$ is not a sub-orbit of $\operatorname{Aut}\left(\Gamma_{1}\right)$. Of course, $\operatorname{Aut}\left(\Gamma_{1}\right)=\operatorname{Aut}\left(\bar{\Gamma}_{1}\right)$ so that $\operatorname{Aut}\left(\bar{\Gamma}_{1}\right)$ is primitive as well. As if $\operatorname{Aut}\left(\Gamma_{1}\right)$ has exactly two sub-orbits, then $\operatorname{Aut}\left(\Gamma_{1}\right)$ is doubly-transitive and hence $\Gamma_{1}=K_{2(p+1)}$ which is not true, Aut $\left(\Gamma_{1}\right)$ has exactly three sub-orbits. Clearly $\mathcal{O}_{3}$ is a sub-orbit of $\operatorname{Aut}\left(\Gamma_{1}\right)$ so that the only sub-orbits of $\operatorname{Aut}\left(\Gamma_{1}\right)$ relative to $v$ are $\mathcal{O}_{1}, \mathcal{O}_{3}$, and $\mathcal{O}_{2} \cup \mathcal{O}_{4}$. Thus the neighbors of $v$ in $\bar{\Gamma}_{1}$ are all contained in one sub-orbit of $\operatorname{Aut}\left(\Gamma_{1}\right)$ relative to $v$. However, one of these directed edges is an edge (as $\bar{\Gamma}_{1}=\Gamma_{2} \cup\left(\bar{K}_{p+1} \prec K_{2}\right)$ ), and so every neighbor of $v$ in $\bar{\Gamma}_{1}$ is an edge. Thus every neighbor of $v$ in $\Gamma_{1}$ is an edge. However, we have already established that $\Gamma_{1}$ is a digraph that is not a graph, a contradiction. Whence $\operatorname{Aut}\left(\Gamma_{i}\right), i=1,2$, are not primitive, and as $\operatorname{SL}(2, p) \leq \operatorname{Aut}\left(\Gamma_{i}\right)$, we have by Lemma 2.1 that $\mathcal{D}_{2}$ is the unique complete block system of $\operatorname{Aut}\left(\Gamma_{i}\right), i=1,2$. Thus (2) holds.

If $\operatorname{fix}_{\operatorname{Aut}\left(\Gamma_{i}\right)}\left(\mathcal{D}_{2}\right)$ is not cyclic, then there exists $1 \neq \gamma \in \operatorname{fix}_{\operatorname{Aut}\left(\Gamma_{i}\right)}\left(\mathcal{D}_{2}\right)$ such that $\gamma(v)=v$ for some $v \in V\left(\Gamma_{i}\right)$. It is then easy to see that $\operatorname{Aut}\left(\Gamma_{i}\right)$ has only three sub-orbits, two of size 1 , and one of size $2 p$, a contradiction. Thus (3) holds.

To establish (4), as $\operatorname{SL}(2, p) / \mathcal{D}_{2}=\operatorname{PSL}(2, p)$ which is doubly-transitive in its action on the blocks (projective points) of $\mathcal{D}_{2}$, we have that $\operatorname{Aut}\left(\Gamma_{i}\right) / \mathcal{D}_{2}$ is doubly-transitive. As $\operatorname{PSL}(2, p) \leq \operatorname{Aut}\left(\Gamma_{i}\right) / \mathcal{D}_{2}$, by $\left[1\right.$, Theorem 5.3] $\operatorname{soc}\left(\operatorname{Aut}\left(\Gamma_{i}\right) / \mathcal{D}_{2}\right)$ is a doubly-transitive nonabelian simple group acting on $p+1$ points. Thus we need only show that $\operatorname{soc}\left(\operatorname{Aut}\left(\Gamma_{i}\right) / \mathcal{D}_{2}\right) \neq$ $A_{p+1}$.

Assume that $\operatorname{soc}\left(\operatorname{Aut}\left(\Gamma_{i}\right) / \mathcal{D}_{2}\right)=A_{p+1}$. Recall that as $p$ is odd, a Sylow 2-subgroup $Q$ of $\mathrm{SL}(2, p)$ is a generalized quaternion group. Furthermore, the unique element of $Q$ of order 2, namely $-I$, is contained is every Sylow 2-subgroup of $\operatorname{SL}(2, p)$ and is semiregular. Observe that as $4 \not(p-1), 4 \mid(p+1)$. Then $Q$ contains an element $\delta$ such that $\delta / \mathcal{D}_{2}$ is a product of $(p+1) / 4$ disjoint 4 -cycles and $\left\langle\delta^{4}\right\rangle=\operatorname{fix}_{\operatorname{Aut}\left(\Gamma_{i}\right)}\left(\mathcal{D}_{2}\right)=\langle-I\rangle$. Let $\delta / \mathcal{D}_{2}=$ $z_{0} \ldots z_{\frac{p+1}{4}-1}$ be the cycle decomposition of $\delta / \mathcal{D}_{2}$. As $\operatorname{soc}\left(\operatorname{Aut}\left(\Gamma_{i}\right) / \mathcal{D}_{2}\right)=A_{p+1}$, there 
exists $\omega \in \operatorname{Aut}\left(\Gamma_{i}\right)$ such that $\omega / \mathcal{D}_{2}=z_{0} z_{1}^{-1} \ldots z_{\frac{p+1}{4}-1}^{-1}$ (note that if $\omega / \mathcal{D}_{2}$ is not an even permutation, then $\delta / \mathcal{D}_{2}$ is not an even permutation, in which case $\operatorname{Aut}\left(\Gamma_{i}\right) / \mathcal{D}_{2}=S_{p+1}$ and $\left.\omega \in \operatorname{Aut}\left(\Gamma_{i}\right)\right)$. Then $\left|\delta \omega / \mathcal{D}_{2}\right|=2$ so that $(\delta \omega)^{2} \in \operatorname{fix}_{\mathrm{Aut}\left(\Gamma_{i}\right)}\left(\mathcal{D}_{2}\right)$. Let $\mathcal{O}_{0}$ be the union of the non-singleton orbits of $\left\langle z_{0}\right\rangle$, and $\mathcal{O}_{1}$ be the union of the non-singleton orbits of $\left\langle z_{1}\right\rangle$ (note that as $p \geq 7, p+1 \geq 8$, so that $(p+1) / 4 \geq 2$ ). Let $D \in \mathcal{D}_{2}$ such that $D \subset \mathcal{O}_{1}$. Then $\left.\delta \omega\right|_{D}$ has order 1 or 2 , so that $\left.(\delta \omega)^{2}\right|_{D}=1$. Thus if $\left.\left.\omega\right|_{\mathcal{O}_{0}} \in \delta\right|_{\mathcal{O}_{0}}$, then $(\delta \omega)^{2} \in \operatorname{fix}_{\mathrm{Aut}\left(\Gamma_{i}\right)}\left(\mathcal{D}_{2}\right)=\langle-I\rangle,(\delta \omega)^{2} \neq 1$, but $(\delta \omega)^{2}$ has a fixed point, a contradiction. Thus $\left.\left.\omega\right|_{\mathcal{O}_{0}} \notin \delta\right|_{\mathcal{O}_{0}}$. Then $H=\left.\langle\delta, \omega\rangle\right|_{\mathcal{O}_{0}}$ has a complete block system $\mathcal{E}$ of 4 blocks of size 2 induced by $\mathcal{D}_{2}$. Furthermore, $H / \mathcal{E}$ is cyclic of order 4 , so that $\operatorname{fix}_{H}(\mathcal{E})$ has order at least 4. Then $\operatorname{Stab}_{H}(v) \neq 1$ for every $v \in \mathcal{O}_{0}$. In particular, $\mathcal{E}$ consists of 4 blocks of size 2 , and $\operatorname{Stab}_{H}(v)$ is the identity on some block of $\mathcal{E}$ while being transitive on some other block. As each block of $\mathcal{E}$ is also a block of $\mathcal{D}_{2}, \operatorname{Stab}_{\operatorname{Aut}(\Gamma)}(v)$ is transitive on some block $D_{v}$ of $\mathcal{D}_{2}$. This then implies that $\operatorname{Stab}_{\text {Aut }\left(\Gamma_{i}\right)}(v)$ has three orbits, two of size one and one of size $2(p+1)-2$, a contradiction.

Corollary 2.3 Let $p=2^{k}-1>7$ be a Mersenne prime. Then there exist exactly two digraphs $\Gamma_{i}, i=1,2$ of order $2^{k+1}$ such that the following properties hold:

1. $\Gamma_{i}$ is an orbital digraph of $\mathrm{SL}(2, p)$ in its action on the set of projective half-points and is not a graph,

2. Aut $\left(\Gamma_{i}\right)$ admits a unique complete block system $\mathcal{D}_{2}$ which consists of $2^{k}$ blocks of size 2 ,

3. $\operatorname{fix}_{\operatorname{Aut}\left(\Gamma_{i}\right)}\left(\mathcal{D}_{2}\right)$ is cyclic of order 2 ,

4. $\operatorname{soc}\left(\operatorname{Aut}\left(\Gamma_{i}\right) / \mathcal{D}_{2}\right)=\operatorname{PSL}(2, p)$ is doubly-transitive,

5. $\Gamma_{i}$ is a Cayley digraph of the generalized quaternion group of order $2^{k+1}$.

Proof. In view of Theorem 2.2, we need only show that $\operatorname{soc}\left(\operatorname{Aut}\left(\Gamma_{i}\right) / \mathcal{D}_{2}\right)=\operatorname{PSL}(2, p)$ and that each $\Gamma_{i}$ is a Cayley digraph of the generalized quaternion group $Q$ of order $2^{k+1}$. As $|\operatorname{SL}(2, p)|=2^{k}\left(2^{k}-1\right)\left(2^{k}-2\right)$, a Sylow 2-subgroup of $\operatorname{SL}(2, p)$ has order $2^{k+1}$, and as $p$ is odd, is isomorphic to a generalized quaternion group of order $2^{k+1}$. As a transitive group of prime power order $q^{\ell}$ contains a transitive Sylow $q$-subgroup [10, Theorem 3.4'], a Sylow 2-subgroup $Q$ of $\mathrm{SL}(2, p)$ is transitive and thus regular. It then follows by [9] that each $\Gamma_{i}$ is isomorphic to a Cayley digraph of $Q$. Furthermore, $\operatorname{Stab}_{\operatorname{Aut}\left(\Gamma_{i}\right) / \mathcal{D}_{2}}(v)$ is of index $2^{k}$ in $\operatorname{Aut}\left(\Gamma_{i}\right) / \mathcal{D}_{2}$. By [5, Theorem 1] we have that either $\operatorname{soc}\left(\operatorname{Aut}\left(\Gamma_{i}\right) / \mathcal{D}_{2}\right)$ is $A_{2^{k}}$ or $\operatorname{PSL}(2, p)$. As by Theorem $2.2, \operatorname{soc}\left(\operatorname{Aut}\left(\Gamma_{i}\right) / \mathcal{D}_{2}\right) \neq A_{2^{k}}$, the result follows.

\section{References}

[1] Cameron, P. J., Finite permutation groups and finite simple groups, Bull. London Math. Soc. 13 (1981) 1-22. 
[2] Cheng, Y., and Oxley, J., On weakly symmetric graphs of order twice a prime, $J$. Comb. Theory Ser. B 42 1987, 196-211.

[3] Dixon, J.D., and Mortimer, B., Permutation Groups, Springer-Verlag New York, Berlin, Heidelberg, Graduate Texts in Mathematics, 163, 1996.

[4] Gorenstein, D., Finite Groups, Chelsea Publishing Co., New York, 1968.

[5] Guralnick, R. M., Subgroups of prime power index in a simple group, J. of Algebra 81 1983, 304-311.

[6] Li, C. H., On isomorphisms of finite Cayley graphs - a survey, Disc. Math., 246 (2002), 301-334.

[7] Marušič, D., and Scapellato, R., Imprimitive Representations of $\operatorname{SL}\left(2,2^{k}\right) J$. Comb. Theory Ser. B 58 1993, 46-57.

[8] Sabidussi, G., The composition of graphs, Duke Math J. 26 (1959), 693-696.

[9] Sabidussi, G. O., Vertex-transitive graphs, Monatshefte für Math. 68 1964, 426-438.

[10] Wielandt, H. (trans. by R. Bercov), Finite Permutation Groups, Academic Press, New York, 1964.

[11] Wielandt, H., Permutation groups through invariant relations and invariant functions, lectures given at The Ohio State University, Columbus, Ohio, 1969.

[12] Wielandt, H., Mathematische Werke/Mathematical works. Vol. 1. Group theory, edited and with a preface by Bertram Huppert and Hans Schneider, Walter de Gruyter \& Co., Berlin, 1994. 